\title{
Active Nonlinear Observers for Mobile Systems
}

\author{
Simon Cedervall and Xiaoming $\mathrm{Hu}$ \\ Optimization and Systems Theory \\ Royal Institute of Technology \\ SE 10044 Stockholm, Sweden
}

\begin{abstract}
For nonlinear systems in general, the observability does not only depend on the initial conditions, but also on the control. This presents an interesting issue: how to design an observer together with the exciting control. In this paper a subproblem in the so called SLAM problem-the relocalization problem is studied, namely, observing the state of a mobile system based on distance sensor readings. An observer is proposed for a car-like robotic system.
\end{abstract}

\section{INTRODUCTION}

For nonlinear systems in general, the observability does not only depend on the initial conditions, but also on the control. This presents an interesting issue: how to design an observer together with the exciting control. This has been a very important issue in the field of active perception in robotics and computer vision [1]. However, study from the systems and control point of view is still lacking [3], [4]. This is witnessed in [5] where it is pointed out that one of the key questions in nonlinear control is "how to design a nonlinear observer for nonlinear systems whose linearization is neither observable nor detectable".

In mobile robotic systems, one typically uses sensors that interact with the environment, such as lasers and video. Based on this background, we consider the following system:

$$
\begin{aligned}
\dot{x} & =f(x)+g(x) u \\
y & =h(x, s) \\
x_{e}(s) & =\phi(s),
\end{aligned}
$$

where $x \in \mathbb{R}^{n}, y \in \mathbb{R}^{p}, u \in \mathbb{R}^{m}$ and $s \in \mathbb{R}^{q} . x_{e}(s)=\phi(s)$ defines manifolds in $\mathbb{R}^{n}$ that model terrains (walls) in the environment.

In robotics one of the most important problems is the socalled SLAM (simultaneous localization and map building) problem, namely, to reconstruct the map $\phi(s)$ and the state $x$ at the same time. There is a vast literature on it, see for example [2] and the references therein. However, methods for dealing with non-feature based environment and for feedback control design are still lacking.

In this paper we study a subproblem in SLAM: the relocalization problem. Namely, we assume that the map $\phi(s)$ is already given, and we would like to observe the state of the system based on distance sensor readings. Although we will use a specific system model for the problem, the discussion would nevertheless concern some of the fundamental issues about nonlinear observers as aforementioned.

\section{THE SYSTEM SETUP}

We consider an oriented robot in the plane, using the so-called unicycle model. Namely, the state of the system is described by $\left(\left(x_{1}, x_{2}\right), \theta\right) \in \mathbb{R}^{2} \times S^{1}$ and it has two control inputs, the translational velocity $v$ and the angular velocity $\omega$. The governing dynamics are

$$
\begin{aligned}
\dot{x_{1}} & =v \cos \theta \\
\dot{x_{2}} & =v \sin \theta \\
\dot{\theta} & =\omega .
\end{aligned}
$$

Furthermore, we assume that the robot is equipped with two range-measuring sensors, oriented at angles $\varphi_{1}, \varphi_{2}$ with respect to $\theta$. These sensors measure the distances $\rho_{1}, \rho_{2}$, to some smooth curve $r: S^{1} \rightarrow \mathbb{R}^{2}$ or $r: \mathbb{R} \rightarrow \mathbb{R}^{2}$, along the ray originating at the robot and making an angle $\theta+\varphi_{i}$, $i=1,2$, with the $x_{1}$-axis. Thus we have two outputs for the system

$$
\begin{aligned}
& y_{1}=\rho_{1} \\
& y_{2}=\rho_{2}
\end{aligned}
$$

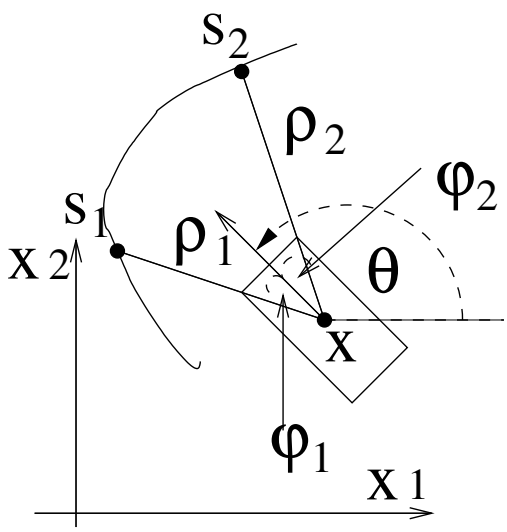

Fig. 1. The system setup

In this paper we assume that the curve $r$ is closed, i.e. $r: S^{1} \rightarrow \mathbb{R}^{2}$, and that it encircles a convex domain $D$. The robot is restricted to move in the interior of this domain, and thus the outputs $\rho_{1}, \rho_{2}$ are well defined.

Our problem is to construct an observer for the full state of the robot. It is easy to see that this problem is equivalent to the reconstruction of orientation $\theta$ and the two parameter values $s_{1}, s_{2} \in S^{1}$ corresponding to the points on the curve 
measured against. In order to do this we define a (new) state variable $p=\left(s_{1}, s_{2}, \theta\right) \in S \subset T^{3}$, which we call the parameter configuration. Since we will be concerned with local properties only, we can consider $p$ as an element of $\mathbb{R}^{3}$.

For the configuration $(x, \theta) \in \mathbb{R}^{2} \times S^{1}$, the distances $\rho \in \mathbb{R}^{2}$ and the parameters $s \in \mathbb{R}^{2}$, we have the basic geometrical relationship

$$
x+\rho_{i} R_{\theta} R_{\varphi_{i}} e_{1}=r\left(s_{i}\right),
$$

and differentiating,

$$
v R_{\theta} e_{1}+\dot{\rho}_{i} R_{\theta} R_{\varphi_{i}} e_{1}+\rho_{i} \omega R_{\theta} R_{\varphi_{i}} e_{2}=r^{\prime}\left(s_{i}\right) \dot{s}_{i} .
$$

Here we use the notation $e_{1}=(1,0)^{T}, e_{2}=(0,1)^{T}$, and

$$
R_{\alpha}=\left[\begin{array}{cc}
\cos \alpha & -\sin \alpha \\
\sin \alpha & \cos \alpha
\end{array}\right] \text {. }
$$

For future use, we also define $M=R_{\pi / 2}$, and note that $\partial R_{\alpha} / \partial \alpha=M R_{\alpha}=R_{\alpha} M, M e_{1}=e_{2}, M e_{2}=-e_{1}$.

Multiplying (10) with $e_{2}^{T} R_{-\varphi_{i}-\theta}$ from the left gives

$$
\dot{s}_{i}=\frac{-v \sin \varphi_{i}+\rho_{i} \omega}{r^{\prime}\left(s_{i}\right)^{T} R_{\theta} R_{\varphi_{i}} e_{2}},
$$

and multiplying (2) with $r^{\prime}\left(s_{i}\right)^{T} M$, one obtains

$$
\dot{\rho}_{i}=\frac{-v r^{\prime}\left(s_{i}\right)^{T} R_{\theta} e_{2}+\rho_{i} \omega r^{\prime}\left(s_{i}\right)^{T} R_{\theta} R_{\varphi_{i}} e_{1}}{r^{\prime}\left(s_{i}\right)^{T} R_{\theta} R_{\varphi_{i}} e_{2}} .
$$

The above expressions are well-defined except when $r^{\prime}\left(s_{i}\right)^{T} R_{\theta} R_{\varphi_{i}} e_{2}=0$, which occurs when a sensor measures tangentially to the wall. In the convex setting we have assumed, this will not happen.

Using only the values of $\rho_{1}$ and $\rho_{2}$ it is easy to see that we will, in general, only be able to determine the configuration of the robot up to some curve in $\mathbb{R}^{2} \times S^{1}$. One can picture this by following a planar curve with the tips of two extended fingers. We call this curve the statically unobservable submanifold. This fact in particular suggests that the nonlinear system defined by (4) and (7) is not always observable.

By also taking $\dot{\rho}$ into account, we find a similarly unobservable submanifold, also one-dimensional. The theoretical basis for active nonlinear observer design as we will show later is that under certain conditions the two different submanifolds are not parallel at the actual parameter configuration of the robot, and hence the system ought to be locally observable.

\section{THE STATICALLY UNOBSERVABLE SUBMANIFOLD}

For a general parameter vector $p=\left(s_{1}, s_{2}, \theta\right)$ and two distances $\rho_{1}, \rho_{2}$, we define

$$
\begin{aligned}
z_{1}(p) & =r\left(s_{1}\right)-\rho_{1} R_{\theta} R_{\varphi_{1}} e_{1}, \\
z_{2}(p) & =r\left(s_{2}\right)-\rho_{2} R_{\theta} R_{\varphi_{2}} e_{1}, \\
z(p) & =z_{1}(p)-z_{2}(p), \\
V_{z} & =z^{T} z .
\end{aligned}
$$

Clearly, if $\rho_{1}$ and $\rho_{2}$ are measured distances and $p$ the actual parameter configuration of the robot, then $z_{1}=z_{2}=x$, and $z$ and $V_{z}$ vanish. Consider the differential of the map $z: p \mapsto \mathbb{R}^{2}$,

$$
\begin{aligned}
\partial_{p} z & =\left[\begin{array}{lll}
\frac{\partial z_{x}}{\partial s_{1}} & \frac{\partial z_{x}}{\partial s_{2}} & \frac{\partial z_{x}}{\partial \theta} \\
\frac{\partial z_{y}}{\partial s_{1}} & \frac{\partial z_{y}}{\partial s_{2}} & \frac{\partial z_{y}}{\partial \theta}
\end{array}\right] \\
& =\left[\begin{array}{lll}
r^{\prime}\left(s_{1}\right) & -r^{\prime}\left(s_{2}\right) & M\left(r\left(s_{2}\right)-r\left(s_{1}\right)\right)+M z
\end{array}\right]
\end{aligned}
$$

We see that if $z=0$, this is onto (as a linear map from $\mathbb{R}^{3}$ to $\mathbb{R}^{2}$ ) as long as the slope of the curve is not parallel at $r\left(s_{1}\right)$ and $r\left(s_{2}\right)$ and simultaneously perpendicular to the line between these two points. By the Implicit Function Theorem, we then have the following,

Proposition 1: Assume that at a configuration $(x, \theta)$, where $x$ lies in the interior of $D$, the robot measures distances $\rho_{1}, \rho_{2}$ against parameters $s_{1}, s_{2}$. Also assume that $r^{\prime}\left(s_{1}\right)$ and $r^{\prime}\left(s_{2}\right)$ are not parallel or, if they are, not perpendicular to the vector $r\left(s_{1}\right)-r\left(s_{2}\right)$. Then, locally around $\left(s_{1}, s_{2}, \theta\right)$, the equation $z\left(\hat{s}_{1}, \hat{s}_{2}, \hat{\theta}\right)=0$ defines a one-dimensional surface (a curve) which passes through $\left(s_{1}, s_{2}, \theta\right)$. This is called the statically unobservable submanifold.

If we define $d=r\left(s_{2}\right)-r\left(s_{1}\right)$, then the kernel of $\partial_{p} z$ is spanned by

$$
\nu_{z}=\left[\begin{array}{l}
-r^{\prime}\left(s_{2}\right)^{T}(d+z) \\
-r^{\prime}\left(s_{1}\right)^{T}(d+z) \\
r^{\prime}\left(s_{1}\right)^{T} M r^{\prime}\left(s_{2}\right)
\end{array}\right]
$$

which vanishes as the rows of $\partial_{p} z$ become linearly dependent. In general, it might still be possible to find a continuation of the curve on which $V=0$ through higher order terms. A clear case of when this is not possible would be when the sensors measures points on the boundary where there simply is no room to move the robot to a nearby point that would give the same measurements (e.g. measuring along the major axis of an ellipse).

It is also clear that if $V_{z}=0$ (i.e. $z=0$ ) for some $\left(\rho_{1}, \rho_{2}, \hat{s}_{1}, \hat{s}_{2}, \hat{\theta}\right)$ and $z_{1}=z_{2} \in D$, then in the configuration $\left(z_{1}, \hat{\theta}\right)$, the robot measures precisely the distances $\rho_{1}, \rho_{2}$ against the parameters $\hat{s}_{1}, \hat{s}_{2}$. That is, continuation of the level curves from Proposition 1 gives curves of feasible configurations which, from the information given by the measured distances, the robot could occupy.

Then it is clear that the gradient flow

$$
\frac{d \hat{p}}{d t}=-k_{s}\left(\partial_{\hat{p}} z\right)^{T} z(\hat{p}), \quad k_{s}>0
$$

should be included in the observer design. It serves to localize the parameter configuration to the submanifold of feasible configurations.

Next, we will discuss what can be done in the statically unobservable direction. 


\section{ACTIVE RELOCALIZATION AND NONLINEAR OBSERVERS}

It is clear that with any pair of fixed measurements $\left(\rho_{1}, \rho_{2}\right)$, the system will have a one dimensional unobservable submanifold. Thus it is necessary to design controls such that the outputs do not remain constant. In this section we will first discuss what are the constraints we need impose on the motion in order to make the system observable.

Assuming that we have obtained a point on the curve of feasible configurations by some optimization method, we would now like to localize the actual configuration of the robot by finding a point on the curve where the estimated output flow (time derivatives of the measured distances) coincide with the actual output flow.

From the basic relationship

$$
x+\rho_{i} R_{\theta} R_{\varphi_{i}} e_{1}=r\left(s_{i}\right),
$$

we obtain

$$
r^{\prime}\left(s_{i}\right)^{T} R_{\theta}\left(v e_{2}+\dot{\rho}_{i} R_{\varphi_{i}} e_{2}-\rho_{i} \omega R_{\varphi_{i}} e_{1}\right)=0
$$

by first differentiating with respect to time and then multiplying by $r^{\prime}\left(s_{i}\right)^{T} M$ from the left. Now define $F: \mathbb{R}^{3} \rightarrow$ $\mathbb{R}^{2}$, for given $v, \omega, \rho_{i}, \dot{\rho}_{i}$, by

$$
F\left(s_{1}, s_{2}, \theta\right)=\left[\begin{array}{c}
r^{\prime}\left(s_{1}\right)^{T} u_{1} \\
r^{\prime}\left(s_{2}\right)^{T} u_{2}
\end{array}\right] .
$$

where $u_{1}, u_{2}$ are given by

$$
u_{i}=R_{\theta}\left(v e_{2}+\dot{\rho}_{i} R_{\varphi_{i}} e_{2}-\rho_{i} \omega R_{\varphi_{i}} e_{1}\right) .
$$

Naturally one can use (12) to replace $\dot{\rho}_{i}$.

The differential of $F$ with respect to the parameter vector is given by

$$
\partial_{p} F=\left[\begin{array}{ccc}
r^{\prime \prime}\left(s_{1}\right)^{T} u_{1} & 0 & r^{\prime}\left(s_{1}\right)^{T} M u_{1} \\
0 & r^{\prime \prime}\left(s_{2}\right)^{T} u_{2} & r^{\prime}\left(s_{2}\right)^{T} M u_{2}
\end{array}\right],
$$

The kernel of $\partial_{p} F$, as long as this has full rank, is spanned by

$$
\nu_{F}=\left[\begin{array}{c}
-\left(r^{\prime \prime}\left(s_{2}\right)^{T} u_{2}\right)\left(r^{\prime}\left(s_{1}\right)^{T} M u_{1}\right) \\
-\left(r^{\prime \prime}\left(s_{1}\right)^{T} u_{1}\right)\left(r^{\prime}\left(s_{2}\right)^{T} M u_{2}\right) \\
\left(r^{\prime \prime}\left(s_{1}\right)^{T} u_{1}\right)\left(r^{\prime \prime}\left(s_{2}\right)^{T} u_{2}\right)
\end{array}\right] .
$$

Assume now $F=0$ and $p_{0}=\left(s_{1}, s_{2}, \theta\right)$ are the true parameters and state, then we have

$$
\begin{aligned}
u_{i}\left(p_{0}\right) & =R_{\theta} R_{\varphi_{i}}\left[\begin{array}{c}
v \sin \varphi_{i}-\omega \rho_{i} \\
v \cos \varphi_{i}+\dot{\rho}_{i}
\end{array}\right] \\
& =\left(v \sin \varphi_{i}-\omega \rho_{i}\right) R_{\theta} R_{\varphi_{i}}\left[\begin{array}{c}
1 \\
-\frac{r^{\prime}\left(s_{i}\right)^{T} R_{\theta} R_{\varphi_{i}} e_{1}}{r^{\prime}\left(s_{i}\right)^{T} R_{\theta} R_{\varphi_{i}} e_{2}}
\end{array}\right],
\end{aligned}
$$

or

$$
M u_{i}\left(p_{0}\right)=\frac{\left(v \sin \varphi_{i}-\omega \rho_{i}\right)}{r^{\prime}\left(s_{i}\right)^{T} R_{\theta} R_{\varphi_{i}} e_{2}} r^{\prime}\left(s_{i}\right) .
$$

Hence, we have

$$
\begin{aligned}
\nu_{F}\left(p_{0}\right) & =\frac{\left(v \sin \varphi_{1}-\omega \rho_{1}\right)\left(v \sin \varphi_{2}-\omega \rho_{2}\right)}{\left(r^{\prime}\left(s_{1}\right)^{T} R_{\theta} R_{\varphi_{1}} e_{2}\right)\left(r^{\prime}\left(s_{2}\right)^{T} R_{\theta} R_{\varphi_{2}} e_{2}\right)} \times \\
& \times\left[\begin{array}{c}
\left(r^{\prime \prime}\left(s_{2}\right)^{T} M r^{\prime}\left(s_{2}\right)\right)\left(r^{\prime}\left(s_{1}\right)^{T} r^{\prime}\left(s_{1}\right)\right) \\
\left(r^{\prime \prime}\left(s_{1}\right)^{T} M r^{\prime}\left(s_{1}\right)\right)\left(r^{\prime}\left(s_{2}\right)^{T} r^{\prime}\left(s_{2}\right)\right) \\
\left(r^{\prime \prime}\left(s_{1}\right)^{T} M r^{\prime}\left(s_{1}\right)\right)\left(r^{\prime \prime}\left(s_{2}\right)^{T} M r^{\prime}\left(s_{2}\right)\right)
\end{array}\right] .
\end{aligned}
$$

What we should consider, though, is

$$
\left.\partial_{p} F \nu_{z}\right|_{p_{0}}=\left[\begin{array}{c}
\frac{v \sin \varphi_{1}-\omega \rho_{1}}{r^{\prime}\left(s_{1}\right)^{T} R_{\theta} R_{\varphi_{1}} e_{2}} D_{1} \\
\frac{v \sin \varphi_{2}-\omega \rho_{2}}{r^{\prime}\left(s_{2}\right)^{T} R_{\theta} R_{\varphi_{2}} e_{2}} D_{2},
\end{array}\right]
$$

where

$$
\begin{array}{r}
D_{1}=\left(\left(r^{\prime \prime}\left(s_{1}\right)^{T} M r^{\prime}\left(s_{1}\right)\right)\left(r^{\prime}\left(s_{2}\right)^{T} d\right)+\right. \\
\left.\left(r^{\prime}\left(s_{1}\right)^{T} r^{\prime}\left(s_{1}\right)\right)\left(r^{\prime}\left(s_{1}\right)^{T} M r^{\prime}\left(s_{2}\right)\right)\right), \\
D_{2}=\left(\left(r^{\prime \prime}\left(s_{2}\right)^{T} M r^{\prime}\left(s_{2}\right)\right)\left(r^{\prime}\left(s_{1}\right)^{T} d\right)+\right. \\
\left.\left(r^{\prime}\left(s_{2}\right)^{T} r^{\prime}\left(s_{2}\right)\right)\left(r^{\prime}\left(s_{1}\right)^{T} M r^{\prime}\left(s_{2}\right)\right)\right) .
\end{array}
$$

As long as this is non-zero, the statically unobservable submanifold crosses the dynamically unobservable submanifold transversely. Which implies that we are bound to have at least local observability through $\rho$ and $\dot{\rho}$ or the output flow.

We discuss now what constraints we have to impose on the environment and the control so that $\left.\partial_{p} F \nu_{z}\right|_{p_{0}}$ does not vanish.

We will assume that $\partial_{p} z$ has full rank, so that $\nu_{z}$ can be expressed as (17), which is equivalent to assume

$H$ 1: Denote the range of the sensors by $R_{s}$. There are no two points $s_{1}$ and $s_{2}$ on the curve with $\left\|r\left(s_{2}\right)-r\left(s_{1}\right)\right\| \leq$ $2 R_{s} \sin \frac{\left(\phi_{1}+\phi_{2}\right)}{2}$, such that $r^{\prime}\left(s_{1}\right)$ and $r^{\prime}\left(s_{2}\right)$ are parallel and at the same time perpendicular to $r\left(s_{2}\right)-r\left(s_{1}\right)$.

Should it happen that

$$
\left[\begin{array}{ll}
\sin \varphi_{1} & -\rho_{1} \\
\sin \varphi_{2} & -\rho_{2}
\end{array}\right]\left[\begin{array}{l}
v \\
\omega
\end{array}\right]=0
$$

then $\left.\partial_{p} F \nu_{z}\right|_{p_{0}}$ vanishes completely. Thus we need the following assumption on the control design:

$H$ 2: The exciting control $v, \omega$ should be designed such that $v \sin \varphi_{i}-\omega \rho_{i} \neq 0$, for $i=1,2$.

Having one of the components zero corresponds to moving in such a way that $\dot{s}_{i}=0$ for that sensor. If the sensors are directed to different sides of the steering direction and the control input is not zero, this cannot happen since $\rho_{1}$ and $\rho_{2}$ both are positive.

Assumption $H 2$ has actually suggested a way for designing the active control. Namely, the following criterion can be used:

$$
\max _{|v|,|\omega| \leq k}\left\|\left[\begin{array}{ll}
\sin \varphi_{1} & -\rho_{1} \\
\sin \varphi_{2} & -\rho_{2}
\end{array}\right]\left[\begin{array}{c}
v \\
\omega
\end{array}\right]\right\| .
$$

Under the above assumptions, $\left.\partial_{p} F\right|_{p_{0}}$ will still not have full rank if $r$ has zero curvature $\left(r^{\prime \prime}(s)=0\right)$ at both these points, and $\left.\partial_{p} F \nu_{z}\right|_{p_{0}}$ will vanish if furthermore $r^{\prime}\left(s_{1}\right)$ and $r^{\prime}\left(s_{2}\right)$ are parallel. 
The final way $\left.\partial_{p} F \nu_{z}\right|_{p_{0}}$ can vanish is if

$$
D_{1}=0 \text { and } D_{2}=0 \text {. }
$$

To give a geometrical interpretation of this, consider when $r$ is the unit circle, i.e. $r(\alpha)=R_{\alpha} e_{1}$. Then we have

$$
\begin{aligned}
& \left(r^{\prime \prime}\left(\alpha_{1}\right)^{T} M r^{\prime}\left(\alpha_{1}\right)\right)\left(r^{\prime}\left(\alpha_{2}\right)^{T} d\right)+ \\
& \left(r^{\prime}\left(\alpha_{1}\right)^{T} r^{\prime}\left(\alpha_{1}\right)\right)\left(r^{\prime}\left(\alpha_{1}\right)^{T} M r^{\prime}\left(\alpha_{2}\right)\right) \\
& =e_{2}^{T} R_{\alpha_{2}}^{T}\left(R_{\alpha_{2}} e_{1}-R_{\alpha_{1}} e_{1}\right)+e_{2}^{T} R_{\alpha_{1}}^{T} M R_{\alpha_{2}} e_{2} \\
& =0
\end{aligned}
$$

This corresponds to the observation that in a circular symmetry, neither the measured distances nor the time derivatives thereof change if we displace the robot in the "symmetric direction".

Based on the above discussion, we need to make the last assumption:

$H$ 3: There are no two points $s_{1}$ and $s_{2}$ on the curve with $\left\|r\left(s_{2}\right)-r\left(s_{1}\right)\right\| \leq 2 R_{s} \sin \frac{\left(\phi_{1}+\phi_{2}\right)}{2}$, such that

1. $r^{\prime \prime}\left(s_{1}\right)^{2}+r^{\prime \prime}\left(s_{2}\right)^{2}=0$,

or,

2. $D_{1}=0, D_{2}=0$.

\section{Design of observer}

Under the above assumptions, we propose the following observer

$$
\frac{d \hat{p}}{d t}=-k_{s}\left(\partial_{\hat{p}} z\right)^{T} z(\hat{p})-k_{a}\left(\partial_{\hat{p}} F\right)^{T} F(\hat{p}),
$$

where $k_{s}, k_{a}>0$ are some suitably chosen feedback gains.

\section{CONVERGENCE ANALYSIS}

In this section we show that (25) has locally bounded error around $p_{0}(t)$, namely the true parameters and state trajectory.

Proposition 2: Under Assumptions H1-3, by using the observer (25), the estimation error is bounded locally and the bound can be made arbitrarily small by tuning $k=k_{s}=$ $k_{a}$, provided the control and its rate of change are bounded.

\section{Proof}

It is easy to see that the true parameters and state trajectory $p_{0}(t)$ is indeed an equilibrium for (25), since $z\left(p_{0}(t)\right)=0$ and $F\left(p_{0}(t)\right)=0$.

Now we define a candidate Liapunov function as

$$
V(\hat{p}, t)=z^{T}(\hat{p}) z(\hat{p})+F^{T}(\hat{p}) F(\hat{p}) .
$$

Obviously $V$ is locally positive definite around $p_{0}(t)$ if $H 1$ 3 are satisfied.

It is straight forward that

$$
\begin{aligned}
\dot{V} & =\frac{\partial V}{\partial \hat{p}} \frac{d \hat{p}}{d t}+\frac{\partial V}{\partial t} \\
& =-2 k\left\|\left(\partial_{\hat{p}} z\right)^{T} z+\left(\partial_{\hat{p}} F\right)^{T} F\right\|^{2}+f_{s}(t)+f_{a}(t) .
\end{aligned}
$$

By our assumptions the first item in the above equation is locally negative definite, otherwise the two submanifold would not intersect transversely. And both $f_{s}(t)$ and $f_{a}(t)$ are bounded. Therefore one can tune the error by tuning $k_{s}$ and $k_{a}$.

\section{Simulation}

In this section we show some Matlab simulation results. In the simulation setup, the robot is placed inside an ellipse shaped closed wall.

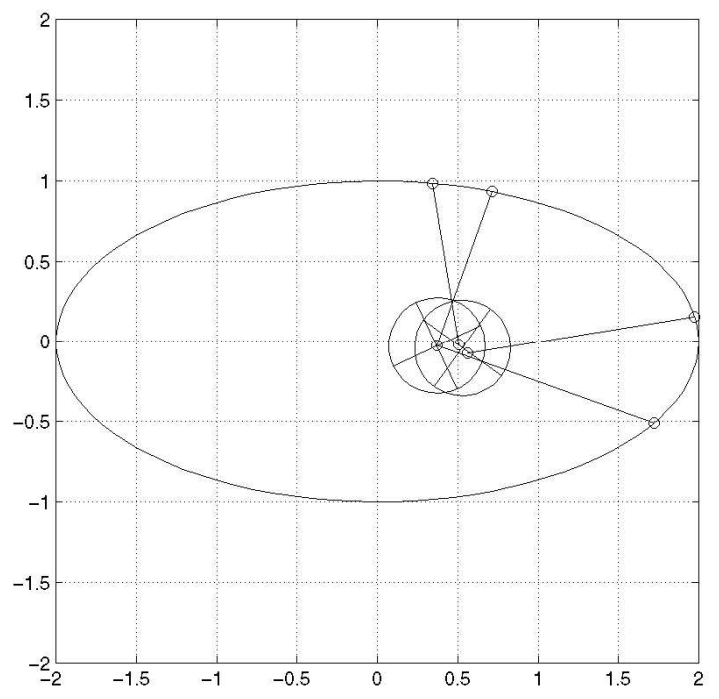

Fig. 2. The initial estimation

Figure 2 shows the initial guess of the state versus the true state. Figure 3 shows the estimation of the state is

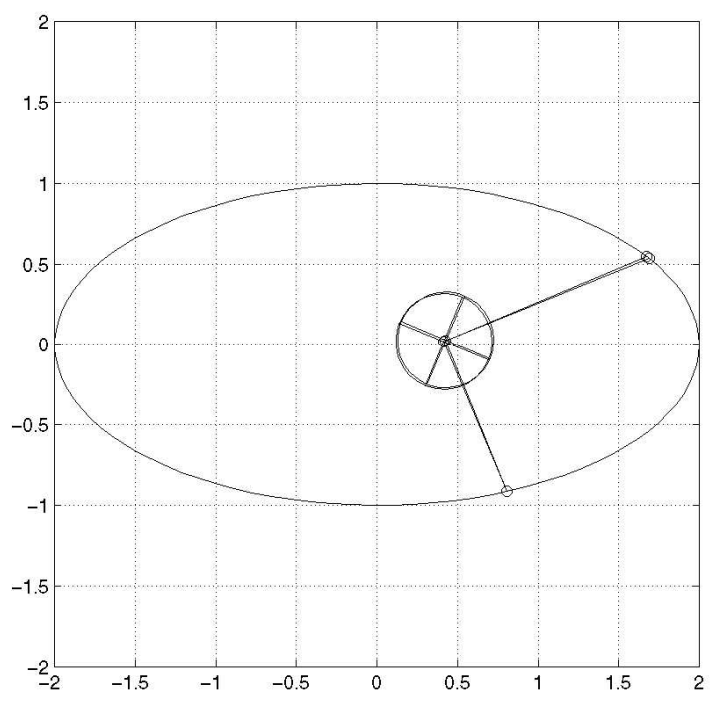

Fig. 3. The final estimation

converging to the true state. Figure 4 shows the history of estimation errors. 

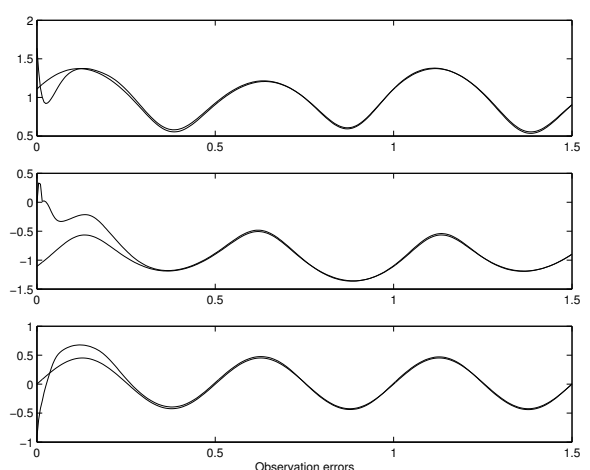

Fig. 4. The observation errors

\section{REFERENCES}

[1] J.-O. Eklundh, T. Uhlin, P. Nordlund, and A. Maki, Active vision and seeing robots, in The 7th Symp. on Robotics Res. (G. Giralt and G. Hirzinger, eds.), Lecture Notes in Computer Science, pp. 416-427, Springer Verlag, Berlin, 1996.

[2] M. Dissanayake, P. Newman, S. Clark, H. Durrant-Whyte and M. Csorba, A solution to the simultaneous localization and map building (SLAM) problem, IEEE Transactions on Robotics and Automation, Vol: 17 (3), Jun 2001

[3] T. Ersson and X. Hu, Implicit Observers and Active Perception, in the proc. of IROS 2001

[4] X. Hu and T. Ersson, Active state estimation of nonlinear systems, submitted to Automatica.

[5] Wei Lin, John Baillieul and A. Bloch, "Call for papers for the special issue on new directions in nonlinear control", IEEE Transactions on Automatic Control 47, no.3, 2002, 543-544. 